\title{
A Survey on to Enhancement the Click Stream of Website using GRC Constraints in Web Personalize Clustering Approach
}

\author{
Ganga Singh ${ }^{1}$, Harsh Pratap Singh ${ }^{2}$, Kailash Patidar ${ }^{3}$ \\ M. Tech. Scholar, Computer Science, Sri Satya Institute of Technology, Sehore (M.P.), India ${ }^{1}$ \\ Assistant Professor, CSE, Sri Satya Institute of Technology, Sehore (M.P.), India ${ }^{2,3}$
}

\begin{abstract}
In the current trends every organization manages work and its data online. Even though e-Commerce website maintaining data online in a distributed form. Online approach is very useful to collaborate with consumer and seller without any dependency of place and time. Every consumer can select product with any brand without wait for a time and produce the order for purchasing. Most of purchasing the product is done by using the website that produce some navigational or access pattern. This access pattern is utilized to produce some access rules. The projected Constraint based Closed Sequential Pattern Mining by using Self-Organizing Map Clustering (CBCSPMSC)approach principal comprises specific profile and GRC constraints for filtration of data among the duration and occurrence of article gap. Now applying closed pattern method for underrates the number of rules generation and execution time. At last SOM clustering method is implemented so that each item belongs the cluster for partial database scan not whole data with fewer execution time.
\end{abstract}

Keywords: Compactness, Data Stream, Data Mining, Web Usage Mining ,Gap, Personalization, Closed Pattern, Sequential Pattern Mining, NN-SOM Clustering

\section{INTRODUCTION}

The prevalent source of distributing is the World Wide Web (WWW) is exceptionally rich wellspring of data gathering. It understanding information is trouble-some on the grounds that distribution on the web is generally unorganized. Web mining is likewise learning extraction systems which find get to designs from the web. It is separated into three sections, a) web utilization mining, b) web structure mining and c) web content mining. The generally utilized information mining calculations are Association Rule Mining (ARM), Sequential Pattern Mining, Clustering, and Classification. An ARM system is utilized to ascertain the standards between things found in an transaction database. With regards to web utilization mining an exchange is a gathering of website page gets to with a thing being a solitary pages get to. The issue of finding successive examples is that of finding between exchange examples with the end goal that the nearness of a lot of things is trailed by another thing in the time-stamp requested exchange set. The information mining calculations are utilized to produce the affiliation rule between the things, consecutive example of access of things, and grouping of things.

Web Usage Mining (WUM) is the use of information mining methods to extensive Web information vaults so as to deliver results that can be utilized in the structure undertakings and improves reaction time. Clustering analysis is utilized to discover those things that have comparable qualities and gathering into it. It deals with the gathering of client data or information from Web server logs. It additionally can encourage the improvement and execution of future showcasing techniques. It powerfully support or changing a specific site dependent on a guest on an arrival visit. A use of existing information mining calculations, for example revelation of affiliation rules or consecutive examples, the general errand isn't one of basically adjusting existing calculations to new information.

The WUM procedure is a document which having contribution from web client conduct as a client session records that gives a definite bookkeeping of who got to the site. It is likewise having the data simply like what pages were asked for and in what request, and to what extent each page was seen. A client session is a period interim where a web client gets to the pages that happen amid a solitary visit to a site. The web client's entrance related all the data contained in a crude web server log. It doesn't dependably speak to a client session record for various reasons. So that specifically data changes over into unthinkable structure and after that apply data mining procedure. In the wake of getting result it creates some significant and valuable data. The Compactness-Constrains is connected to discover the record between the dates with the goal that it can create most recent and intriguing quality example. This limitation needs that the consecutive examples in the sequence database must have the property with the end goal that the time-stamp difference 
(variance of days) between the first and the previous transactions in a found successive example must not be more noteworthy than given period. For Example , if our succession database is from 31/12/2016 to 31/12/2017. The Recency-limitation characterizes when the last transation occurs. This requirement is expressed by giving a recency least help (r_min_sup), which is the quantity of days left from the beginning date of the sequencet database. For instance, if our succession database is from 31/12/2016 to 31/12/2017 and on the off chance that we set $r_{-}$min_sup = 166, at that point the recency imperative guarantees that the last transaction of the found example must happen following 31/12/2016+166 days implies till 15/06/2017.

Gap Constraint is the contrast between two things in transaction events. The hole limitation applies limit on the division of two sequential transaction of found examples. For Example the grouping $\mathrm{S}=\mathrm{ACACBCB}$ and subsequence $\mathrm{S} 0=\mathrm{AB}$, there are 4 event of S0 in S: (A1, B5) (A1, B7), (A3, B5), (A3, B7). Here just the event (A3, B5) satisfy the 1hole requirement. In this way, the subsequence $S 0$ satisfies the 1-hole requirement since no less than one of its events does. No event of S0 satisfies the 0-hole requirement thus S0 comes up short the o-hole limitation. Self-Organizing Map is a Neural Networking Clustering strategy which doles out everything to bunch id. It is utilized to discover the quantity of group dependent on property separate an incentive between of item.

\section{LITERATURE REVIEW}

In 2013, Omar Zaarour, Mohamad Nagi [14] proposed an improvement of the web log mining methodology and to the expectation of online navigational example. It proposed for session distinguishing proof utilizing a refined time-out based heuristic. After identify the navigational example by utilizing a particular thickness based calculation. Presently at long last, another proposed technique for effective online expectation is likewise suggested for pertinence.

In 2016, Doddegowda B J, G T Raju, Sunil Kumar S Manvi[16] having way to deal with customize the data accessible on the Web as indicated by client prerequisites. It changes the data/administrations conveyed by a Web to the necessities of every client or gathering of clients to locate the personal conduct standards.

In 2016, Minubhai Chaudhari and Chirag Mehta [17] proposed a prefix-span calculation with GRC imperatives which produces successive examples by utilizing prefix anticipated example development approach. It utilizes hole, conservativeness and recency limitations amid successive example mining process. The hole imperative applies limit on the partition of two continuous exchanges of found examples, recency limitation makes examples to rapidly adjust the most recent practices and minimization requirement set aside a few minutes ranges for the found examples.

In 2016, Fan Muhan, Shao Sujie, and RuiLanlan[18] proposes a technique for mining the incessant shut examples in a sliding window to catch data auspicious and precisely when new information stream arrives. Here every fundamental window is utilized to store the Closed Pattern-tree in sliding window refreshes which is gradually refreshed and erase the rare or unclosed designs.

In 2017, H. Ryang [19] propose a novel calculation for discovering high utility examples in the rundown structure over information streams based on a sliding window mode. It keep away from the age of hopeful examples to improve the proficiently works in complex unique frameworks.

In 2017 Bing Zhang and Guoyan Huang [20] proposed a way to deal with productively mine successive example utilizing persuasive capacities dependent on programming execution grouping. It can happen on different occasions in a follow, which prompts surprising expense of time and outrageous multifaceted nature of the exploration.

In 2018 PasiFranti [21] proposed arbitrary swap calculation is additionally exceptionally supportive to unravel the bunching by utilizing a succession the executives of model swaps strategy.

\section{PROBLEM DESCRIPTION}

Frequent sequence mining is an imperative part identified with web information and now yet a testing data mining work. The incessant sequence mining has turned into an essential segment of numerous forecast or proposal systems. The online stores each time need client's next thing forecast as pages prone to visit. It additionally prefers to purchase together which items. The current calculations utilized for continuous sequence mining could be ordered either as precise or surmised calculations. Exact continuous grouping mining calculations more often than not read the entire database a few times, and on the off chance that the database is extensive, at that point visit succession mining isn't good with restricted accessibility of PC devices and ongoing imperatives. So the issues in the present situation are -

1) Web information packets isn't utilized some contingent parameters simply like profile requirement (Income, Age, and Experience and so on.) which support as restrictive parameter for segment of web information. 
2) Many past consecutive mining calculations demonstrate no impression of significance of pages while each page has distinctive significance. So the current strategies perform reaction time is additionally moderate. Site required sensible inexact techniques for examining information where the calculation speed could easily compare to the exactness.

3) Every time the entire database examines for looking through the continuous example not fractional database. At the season of program execution number of cluster required as an information parameter.

\section{PROPOSED APPROACH}

The proposed work utilizes GRC constraints to gather the right information after that apply the Profile coordinating dependent on matching of profile property. The proposed methodology is utilized to improve the web reaction for the online navigational pattern forecasting. It is demonstrating the blend of two methodologies Closed Sequential Pattern and Self Organizing Map Clustering for finding regular sequence traversal pattern with GRC requirement. Here each thing has a place one cluster for incomplete information scan .So right now this cluster information tree having the site pages of site in corresponding sessions can get to partially. This research utilizing a novel methodology Profile based Constraint based Closed Sequential Pattern Mining utilizing SOM Clustering (CBCSPMSC). It is utilized as a pattern examination to distinguish client's examples during the time spent web use mining. It relies upon the execution of the grouping of the measure of solicitations. Here the proposed methodology utilizing SOM grouping for gets to the incomplete information of web information. The information is gathered from the site www.getglobalindia.org as web $\log$. Each web $\log$ is having 13 parameter segments. IP-Address, Web Browser, Version, OS and so forth. So at preprocessing the weblog is channel by chosen characteristics and gets just required information after that gathering the required data as indicated by client session-wise for finding the client conduct. In the following stage the info bolster assemble by client utilizing Interface, in the event that the thing support is more noteworthy than and equivalent to given help, at that point it delivers the continuous thing utilizing pruning system of the thing.

Proposed CBCSPMSC Algorithm Description - The accompanying Fig. 1 demonstrates that the procedure of CBCSPMSC calculation which produce valuable shut successive example utilizing web information.

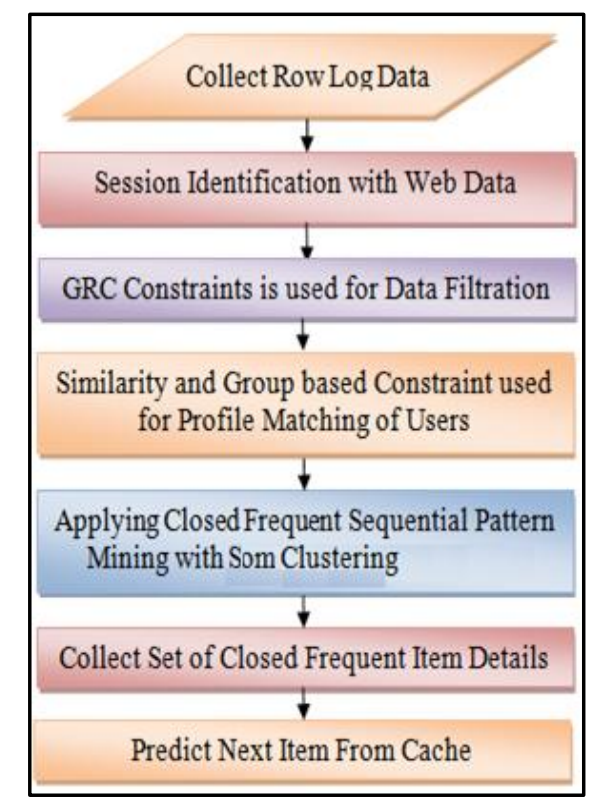

Fig. 1: The Progression of Proposed Algorithm

\section{Proposed Algorithm Description}

Step 1: Collection of web navigation history of website.

Step 2: Apply pre-processing techniques to remove noise from web log data and also convert into proper format.

Step 3: Now choose the GRC Constraint for filter the correct data and then apply attributes of profile for similarity matching and input the support value. So here it is matching the attribute similarity to other user navigation pattern group wise. It is also find the frequent pattern using given support threshold value in the model.

Step 4: Now generate rules using closed sequential pattern of web data set.

Step 6: For clustering the web data set, first find the smallest probability value and after that merge these two by taking smallest value in the form of cluster. 


\title{
International Journal of Advanced Research in Computer and Communication Engineering
}

\author{
Vol. 8, Issue 3, March 2019
}

Step 7: Select different size of web data set and generate the rules.

Step 8: Put those item in the cache which are having higher frequency.

Step 9: For the next item prediction put some items in the cache which having higher frequency. Sometimes if next item not in the cache so that it scan the related item from the cluster web data set not the whole data set.

\section{CONCLUSION}

In this exploration a novel methodology CBCSPMSC is proposed for finding close successive patterns and sweep just fractional web information for next item prediction. It filtering expansive web information by coordinating users profile similarity dependent on certain attributes. It is having least help and everything has a place with bunch so partial web information is examine. Close incessant pages are exceptionally less and helpful principles as grouped by SOM clustering. The real confines of the customary methodology for mining designs is that weight of each page is refreshed physically, however by proposed strategy it is refreshed naturally utilizing web services. On the off chance that web information size is 6050 and support is $10 \%$, at that percentage improvement in execution time (in ms) is $2.41 \%$. Also on the off chance that the help is $40 \%$, at that percentage improves in execution time (in ms) is $7.42 \%$. It perform quick reaction and precise outcome on account of this it is persuasive sufficient to do hugely figuring expensive tasks in a generally short measure of time for finding next page forecast. In future work, other data mining algorithm can be actualized in cloud to productivity handle huge information of numerous Hospital site in appropriated condition for finding

\section{REFERENCES}

[1]. R.A. Agrawal and R. Srikant, "rapid Algorithms for Association Constraints of Mining in Large Database ", in Proc. Int. Conf. Very Large Data Bases, pp. 487-499, 1994

[2]. M.N.P.Rastogi R.Garofalkis "SPIRIT : Sequential Pattern Mining with Regular Expression Constraints", In Proceedings of 25th VLDB Conference, pp. 223-234, San Francisco, California, 1999.

[3]. N. J. Zak , "SPADE: An Efficient Algorithm for Mining Frequent Sequences", Machine Learning Journal, Vol. 42, Issue (1-2), pp. 31-60, 2001.

[4]. J P, Jiawei H\&H Pinto, "Prefix-Span: Mining Sequential Patterns Efficiently by Prefix- Projected Pattern Growth", In Proceedings of $12^{\text {th }}$ International Conference on Data Engineering, pp.215-224, Heidelberg, Germany, 2001.

[5]. F. J., Kumar B., and Lieuwen D., "Web-Views: Accessing Personalized Web Contents and Service", In Proceedings of the Tenth International World Wide Web Conference, 2001

[6]. Antunes, A. L. Oliveira, "Generalization of Pattern-growth Methods for Sequential Pattern Mining with Gap Constraints", Machine Learningand Data Mining in Pattern Recognition, Third International Conference, MLDM 2003, Leipzig, Germany, July 5-7, 2003, Proceedings 2003.

[7]. J. Han, J. Pei, Y. Yin, and R. Mao, "Mining Frequent Patterns without Candidate Generation: A Frequent-Pattern Tree Approach", Data MiningKnowledge Discovery, vol. 8, no. 1, pp. 53-87, 2004

[8]. J. Pei et al., "Mining Sequential Patterns by Pattern-Growth: The PrefixSpan Approach", IEEE Trans. Knowledge Data Eng., vol. 16, no. 11, pp. 1424-1440,Nov. 2004.

[9]. Yen-Liang Chen, Ya-Han Hu, "The Consideration of Recency and Compactness in Sequential Pattern Mining", In Proceedings of the second workshop onKnowledge Economy and Electronic Commerce, Vol. 42, Iss. 2 ,pp. 1203-1215, 2006.

[10]. T.P. Hong, C.W. Lin, and Y.L. Wu, "Incrementally Fast Updated FrequentPattern Trees", Expert System Application, vol. 34, no. 4, pp. 24242435, May 2008

[11]. Krzysztof D., Wojciech K., Marcin S., "Effective Prediction of Web User Behaviour with User-Level Models", Fundamental Informatics , IOS Press, Vol. 89, No. 2-3, pp. 189, 2008.

[12]. K. R. Suneetha, Dr. K. R. Krishnamoorthy, "Identifying User Behavior by Analyzing Web Server Access Log File”, IJCSNS International Journal of Computer Science and Network Security, Vol. 9, No.4, pp. 327, 2009.

[13]. D.K.Jha, A. Rajput, M.Singh. \&Archana Tomar, (2010) "An Efficient Model for Information Gain of Sequential Pattern from Web Logs based on Dynamic Weight Constraint", IEEE International Conference on Computer Information Systems and Industrial Management.

[14]. Omar Zaarour, Mohamad Nagi, "Effective Web Log Mining and Online Navigational Pattern Prediction”, ELSEVIER, 2013.

[15]. Jerry Chun, W. G., Tzung Pei Hong, "Efficiently Maintaining the Fast Updated Sequential Pattern Trees With Sequence Deletion", IEEE Access - The Journal for Rapid open access publishing, Vol. 2, pp. 1374-1383, 2014

[16]. Doddegowda B. J., G. T. Raj, Sunil Kumar, "Extraction of Behavioral Patterns from Pre-processed Web Usage Data for Web Personalization", IEEE International Conference on Recent Trends in Electronics Information Communication Technology, pp. 494-498, 2016.

[17]. Min Cha, Chi Mehta, "Extension of Prefix Span Approach with GRC Constraints for Sequential Pattern Mining", International Conference on Electrical, Electronics, and Optimization Techniques, pp. 2496-2498, 2016

[18]. Fan Muhan, S S, Rui-Lanhan, "A Mining Algorithm for Frequent Closed Pattern on Data Stream based on Sub Structure Compressed in Prefix Tree", IEEE Proceedings of CCIS, pp. 434-439, 2016.

[19]. H. Ryang and U. Yun., "Efficient High Utility Pattern Mining for Establishing Manufacturing Plans with Sliding Window Control", IEEE Expert Systems with Applications, Vol. 57, pp. 214-231, 2017

[20]. B.Z., Guoyan Huang, Haitao He, J.R., "To Approach Mining Influential Functions Based on Software Display Sequence", International Journal of Engineering and Technology, Vol. 11, Issue 2, pp. 48-54, 2017.

[21]. Pasi Franti., "Efficiency of Random Swap Clustering”, Journal of Big Data, Springer, Vol. 5, Issue 13, pp. 2-29, 2018

[22]. LiwinPeng. , Yongguo Liu, "Properties Selection and Overlapping Clustering-Based Multi-label Classification Model", Hindawi, pp. 1-13, 2018.

[23]. Dr. S.K. Jayanthi, C. Kavipriya, "Clustering Approach for Classification of Research Articles based on Keyword Search”, IJARCET, Vol. 7, Issuse 1, pp. 86-90, 2018. 